\title{
Decision-Feedback Multiuser Detection in Multicell Multicarrier DS-CDMA Systems with/without BS Cooperation
}

\author{
Xiaojie Ju and Youguang Zhang \\ School of Electronic and Information Engineering \\ Beihang University, Beijing 100083, China \\ Email: juxiaojie798@hotmail.com,zhangyouguang@vip.sina.com
}

\author{
Lie-Liang Yang \\ School of ECS, University of Southampton \\ SO17 1BJ, United Kingdom \\ Tel: 0044-(0)23-8059 3364, Email: 1ly@ecs.soton.ac.uk
}

\begin{abstract}
In this contribution, we investigate the signal detection in the multicarrier direct-sequence code-division multiple-access (DS-CDMA) systems employing both time (T)-domain and frequency (F)-domain spreading, which are referred to as the TF/MC DS-CDMA systems. A multicell scenario with universal frequency reuse is considered, where signals experience frequency-selective Rayleigh fading. A decision-feedback multiuser detector (MUD), which represents the extension of a so-called receiver multiuser diversity aided multi-stage minimum mean-square error MUD (RMD/MS-MMSE MUD), is employed to cope with both the intracel multiuser interference (MUI) and intercell interference (ICI), when basestation (BS) cooperation is or is not assumed. Furthermore, when BS cooperation is assumed, symbols detected at different BSs are interchanged and combined to enhance the detection performance. Our studies and performance results demonstrate that the RMD/MS-MMSE MUD constitutes one of the promising multicell processing (MCP) schemes, making it possible for each cell to support heavily overloaded users, even when a frequency-reuse factor one is applied.
\end{abstract}

\section{INTRODUCTION}

Recently, multicarrier code-division multiple-access (MC-CDMA), which combines the benefits of CDMA with the natural robustness to the frequency-selective fading offered by orthogonal frequencydivision multiplexing (OFDM), has attracted a lot of research [1, 2]. In particular, the MC-CDMA employing both time (T)-domain and frequency (F)-domain spreading, referred to as the TF/MC DSCDMA [2], constitutes one of the promising multiuser communication schemes having a range of advantages suitable for the future generations of broadband wireless systems $[2,3]$. However, TF/MC DS-CDMA conflicts multiuser interference (MUI), especially, when the system is operated in overload scenarios, where the number of users supported is higher than the degrees-of-freedom (DoFs), such as spreading factors, number of receive antennas, etc., of the communication system. The situation may become even worse in multicell environments, due to the added intercell interference (ICI) in addition to the intracell MUI. In order to circumvent this problem, a number of approaches have been proposed, among which multiuser detection (MUD) [4] is a highly effective one.

Since Verdu's seminal work, there have been a lot of researches on the MUD, but mainly, for single-cell setup, as evidenced by $[2,4]$ and the references therein. However, the studies on MUD dedicated to multicell setup are limited. In [5], a joint maximum likelihood (JML)MUD has been investigated in the context of the narrowband multicell systems without BS cooperation, where the JML-MUD performs joint detection of the intracell users and some intercell users generating strong ICI.

In multicell systems, when BS cooperation is implemented, an intuitive way is to assume that there is a central signal processing unit (CSPU) and all the BSs are connected to it via backhaul links. In this case, hard-decision data [6] or quantized soft information [7] obtained by the BSs can be sent to the CSPU, where joint detection is carried out in the same way as that in the single-cell. Despite the prominent performance gains over the conventional noncooperative schemes, the centralized schemes are usually impractical and less attractive, due to the demand of impractical complexity for implementation. For this sake, decentralized detection schemes have been proposed and investigated in recent years [7-10]. To be more specific, in [7-9], based on the principles of Turbo decoding, quantized soft information is exchanged iteratively among the neighboring BSs for ICI reconstruction and cancellation. In [10], iterative hard information exchange among BSs is proposed, which is used for improving the ML detection. Although iterative exchange of information is effective in ICI cancellation, the complexity demanded by the multiple rounds of information exchange may impose excessive burden on the backhaul links.

Against the background, in this paper, we propose and investigate the MUD in the multicell TF/MC DS-CDMA systems, which use no BS cooperation or employ the BS cooperation supported by exchanging hard-decision data among the BSs. Specifically, the novel low-complexity MUD scheme, namely the receiver multiuser diversity aided multistage MMSE MUD (RMD/MS-MMSE MUD) previous proposed for single-cell SDMA and DS-CDMA systems [11] is extended to the multicell TF/MC DS-CDMA systems. Furthermore, when BS cooperation is employed, symbols detected at different BSs are interchanged and combined not only to enhance the RMD/MSMMSE MUD's performance, but also to earn some macro-diversity gain provided by the different BSs. Our studies and performance results show that the RMD/MS-MMSE MUD is highly efficient for operation in the multicell environments, where severe ICI and intracell MUI exist. It is capable of attaining promising BER performance, even when each of the cells is heavily overloaded, making the number of users supported per cell higher than the total spreading factor of the TF/MC DS-CDMA system.

\section{MUlticell Time-FreQuenCy-Domain SPREAD MULTICARRIER DS-CDMA SYSTEM MODEL}

We consider the uplink transmission in a three-cell TF/MC DSCDMA system with universal frequency reuse, i.e., the frequency reuse factor is one, the structure of which is depicted in Fig. 1. We assume that each cell has one BS and covers $K$ users, which are referred to as the intracell users. Otherwise, the users in the neighboring cells are called as intercell users. The $K$ users of a cell are randomly located inside the cell. Power control in used, so that the average power received from intracell users is the same, which is normalized to one. The ICI of user $k$ in cell $i$ on BS $m$ is related to a ICI factor (ICIF) $\alpha_{m i}^{(k)}(i \neq m)$, which reflects the combined effects of path loss, shadowing and transmit power of user $k$ in cell $i$. For the sake of simplicity but of illustrating the effect of intercell users, we assume that $\alpha_{m i}^{(k)}$ is subject to the uniform distribution in $[0,1]$. Considering that $\alpha_{m i}^{(k)}$ may be large, for example, when user $k$ is at the border of cell $i$ and $m$, BS $m$ may detect the intercell user $k$ of cell $i$, in order to enhance the system's performance. For this purpose, we classify the intercell users into two classes by comparing their ICIFs with a predefined threshold $0 \leq \lambda \leq 1$. Specifically, the intercell users with their ICIFs exceeding $\bar{\lambda}$ belong to Class-1, while the remaining intercell users belong to Class-2. The Class- 1 intercell users are detected along with the intracell users possibly for different purposes, as specified in our forthcoming discourses. By contrast, the intercell users belonging to Class-2 are simply treated as noise, as their signals are too weak to be detected.

\section{A. Transmitted Signal}

The transmitter schematic for the $k$ th user of cell $i$ in the TF/MC DSCDMA systems is shown in Fig. 2. It includes T-domain spreading, F-domain spreading and multicarrier modulation. For convenience, the main parameters used for characterizing the TF/MC DS-CDMA systems are summarized as follows:

- $T_{b}, T_{c}$ : bit-duration and chip-duration;

- $N_{t}=T_{b} / T_{c}$ : T-domain spreading factor;

- $N_{f}$ : F-domain spreading factor and also the number of the orthogonal subcarriers; 


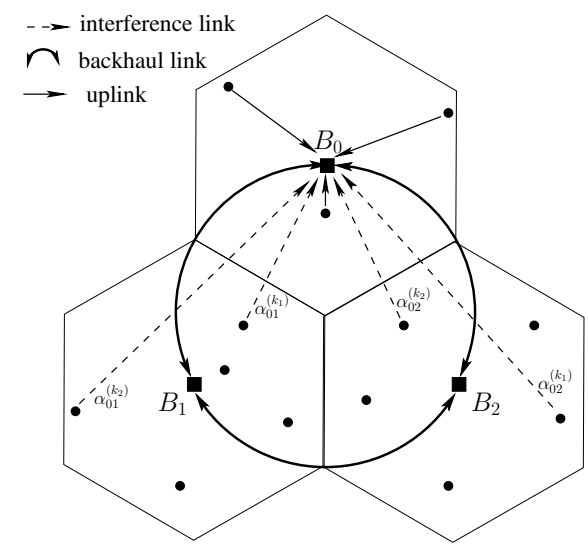

Fig. 1. Illustration of a three-cell system, where each cell has one BS and covers $K$ users.

- $c_{t, i}^{(k)}(t)$ : T-domain spreading waveform of the $k$ th user in cell $i$, formed from the T-domain spreading code $c_{t, i}^{(k)}=$ $\left[c_{t, i}^{(k)}[0], c_{t, i}^{(k)}[1], \ldots, c_{t, i}^{(k)}\left[N_{t}-1\right]\right]^{T} / \sqrt{N_{t}}$, where $c_{t, i}^{(k)}[j] \in$ $\{+1,-1\}$;

- $\boldsymbol{c}_{f, i}^{(k)}$ : F-domain spreading code of the $k$ th user in cell $i, \boldsymbol{c}_{f, i}^{(k)}=$ $\left[c_{f, i}^{(k)}[0], c_{f, i}^{(k)}[1], \ldots, c_{f, i}^{(k)}\left[N_{f}-1\right]\right]^{T} / \sqrt{N_{f}}$, where $c_{f, i}^{(k)}[j] \in$ $\{+1,-1\}$.

As shown in Fig. 2, at the transmitter of the TF/MC DS-CDMA, the binary data stream $x_{i}^{(k)}(t)$ is first spread using a T-domain spreading code $\boldsymbol{c}_{t, i}^{(k)}$. Then, the spread signal is copied to $N_{f}$ parallel branches, where each branch of the signals is multiplied by a corresponding chip value of the F-domain spreading code $\boldsymbol{c}_{f, i}^{(k)}$. Note that, in order to make the subcarriers used by the TF/MC DS-CDMA orthogonal, the spacing between any two adjacent subcarriers is assumed to be $1 / T_{c}$. Following the F-domain spreading, multicarrier modulation is carried out by multiplying each of the $N_{f}$ branches a corresponding subcarrier frequency. Finally, the $N_{f}$ subcarrier signals are summed to form the transmitted signal, which is expressed as

$$
\begin{aligned}
& s_{i}^{(k)}(t)=\sqrt{\frac{2 P}{N_{f}}} \sum_{n_{f}=0}^{N_{f}-1} x_{i}^{(k)}(t) c_{t, i}^{(k)}(t) c_{f, i}^{(k)}\left[n_{f}\right] \\
& \quad \times \Re\left\{\exp \left(j\left[2 \pi f_{n_{f}} t+\phi_{i, n_{f}}^{(k)}\right]\right)\right\}, i=0,1,2
\end{aligned}
$$

where $P$ represents the transmission power of a user, $\left\{f_{n_{f}}\right\}_{n_{f}=0}^{N_{f}-1}$ and $\left\{\phi_{i, n_{f}}^{(k)}\right\}_{n_{f}=0}^{N_{f}-1}$ represent the subcarrier frequencies and the corresponding initial phases, while $\Re\{\cdot\}$ denotes the real part of the complex argument.

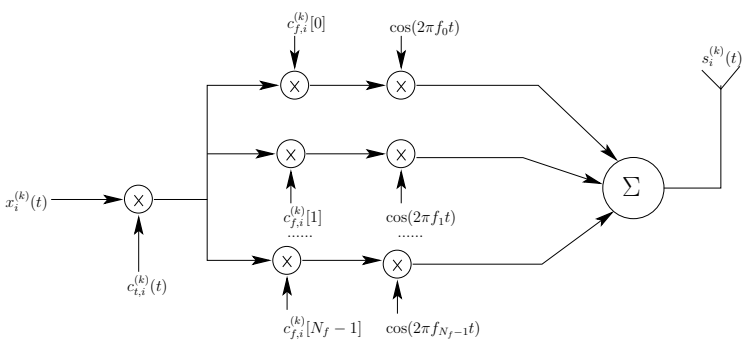

Fig. 2. Transmitter schematic diagram for the $k$ th user of cell $i$ in the TF/MC DS-CDMA system.

B. Channel Model

In this contribution, the channels are assumed the frequencyselective fading channels with $L$ resolvable multipaths. The number of subcarriers of the TF/MC DS-CDMA systems is assumed to satisfy $N_{f} \geq L$, resulting in that each of the subcarriers experiences flat fading [2]. Specifically, for the $k$ th user in the $i$ th $(\mathrm{i}=0,1,2)$ cell, the
T-domain channel impulse responses (CIRs) for the channel from this user to BS $m(m=0,1,2)$ can be expressed as

$$
\overline{\boldsymbol{h}}_{m i}^{(k)}=\left[\bar{h}_{m i, 0}^{(k)}, \bar{h}_{m i, 1}^{(k)}, \ldots, \bar{h}_{m i, L}^{(k)}, 0, \ldots, 0\right]^{T}, m=i
$$

and

$$
\overline{\boldsymbol{h}}_{m i}^{(k)}=\alpha_{m i}^{(k)} \times\left[\bar{h}_{m i, 0}^{(k)}, \bar{h}_{m i, 1}^{(k)}, \ldots, \bar{h}_{m i, L}^{(k)}, 0, \ldots, 0\right]^{T}, m \neq i
$$

respectively, the dimension of which is $\left(N_{f} \times 1\right)$. Besides, $\bar{h}_{m i, l}^{(k)}$ is the complex-valued channel gain, whose amplitude and phase are assumed to obey the Rayleigh distribution and the uniform distribution in $[0,2 \pi)$, respectively.

When the multicarrier modulation and demodulation in the TF/MC DS-CDMA are implemented by the inverse discrete Fourier transform (IDFT) and discrete Fourier transform (DFT) [2], and the cyclicprefixing $(\mathrm{CP})$ is employed to suppress intersymbol interference (ISI), it can be shown that the F-domain CIRs corresponding to (2) and (3) can be expressed as [2]

$$
\boldsymbol{h}_{m i}^{(k)}=\left[h_{m i, 0}^{(k)}, h_{m i, 1}^{(k)}, \ldots, h_{m i, N_{f}-1}^{(k)}\right]^{T}=\sqrt{N_{f}} \mathcal{F} \overline{\boldsymbol{h}}_{m i}^{(k)}
$$

where $\mathcal{F}$ is a normalized DFT matrix satisfying $\mathcal{F} \mathcal{F}^{H}=\mathcal{F}^{H} \mathcal{F}=$ $I_{N_{f}}$, while the factor $\sqrt{N_{f}}$ is applied so that the Parseval's power theorem is satisfied.

\section{Received Signal}

Based on the multicell model as shown in Fig. 1, the TF/MC DSCDMA transmitter structure of Fig. 2 and the channel model as abovedescribed, the received complex low-pass equivalent signal by BS $m$ can be expressed as

$$
\begin{aligned}
R_{m}(t) & =\sum_{i=0}^{2} \sum_{k=0}^{K-1} \sqrt{\frac{2 P}{N_{f}}} \sum_{n_{f}=0}^{N_{f}-1} h_{m i, n_{f}}^{(k)} x_{i}^{(k)}(t) c_{t, i}^{(k)}(t) c_{f, i}^{(k)}\left[n_{f}\right] \\
& \times \exp \left(j\left[2 \pi f_{n_{f}} t+\varphi_{m i, n_{f}}^{(k)}\right]\right)+N(t), m=0,1,2
\end{aligned}
$$

where, in addition to the variables having been stated, the phase $\varphi_{m i, n_{f}}^{(k)}$ takes into account of both the initial subcarrier phase and the phase introduced by the channel, while the complex low-pass equivalent Gaussian process $N(t)$ has a mean of zero and a singlesided power spectral density of $N_{0}$ per dimension.

At BS $m$, the signal of (5) is passed through $N_{f}$ chip waveform matched-filters (MFs) [2], each of which for one subcarrier. It can be shown that, after the MFs, the discrete observations obtained by BS $m$ can be expressed as

$$
\boldsymbol{y}_{m}=\sum_{i=0}^{2} \sum_{k=0}^{K-1} \boldsymbol{C}_{t, i}^{(k)} \boldsymbol{H}_{m i}^{(k)} \boldsymbol{c}_{f, i}^{(k)} x_{i}^{(k)}+\boldsymbol{n}_{m}, m=0,1,2
$$

where $\boldsymbol{y}_{m}$ is a $N_{f} N_{t}$-length observation vector, $\boldsymbol{C}_{t, i}^{(k)}$ is a $\left(N_{f} N_{t} \times\right.$ $N_{f}$ ) matrix that can be expressed as $\boldsymbol{C}_{t, i}^{(k)}=\boldsymbol{I}_{N_{f}} \otimes \boldsymbol{c}_{t, i}^{(k)}$, where $\otimes$ represents the Kronecker product operation. In (6), $\boldsymbol{H}_{m i}^{(k)}$ is a $\left(N_{f} \times\right.$ $N_{f}$ ) diagonal matrix containing the channel gains of the corresponding $N_{f}$ subcarriers, which is

$$
\boldsymbol{H}_{m i}^{(k)}=\operatorname{diag}\left\{h_{m i, 0}^{(k)}, h_{m i, 1}^{(k)}, \ldots, h_{m i, N_{f}-1}^{(k)}\right\}
$$

Finally, $\boldsymbol{n}_{m}$ is a $N_{f} N_{t}$-length Gaussian noise vector distributed with a mean of zero and a covariance matrix $\sigma^{2} \boldsymbol{I}$, where $\sigma^{2}=1 / \gamma_{b}$ with $\gamma_{b}$ representing the average signal-to-noise ratio (SNR) per bit.

When expressed in a compact form, (6) can be written as

$$
\boldsymbol{y}_{m}=\sum_{i=0}^{2} \boldsymbol{C}_{t, i} \boldsymbol{H}_{m i} \boldsymbol{C}_{f, i} \boldsymbol{x}_{i}+\boldsymbol{n}_{m}, m=0,1,2
$$


where, by definition,

$$
\begin{aligned}
& \boldsymbol{C}_{t, i}=\left[\boldsymbol{C}_{t, i}^{(0)}, \boldsymbol{C}_{t, i}^{(1)}, \ldots, \boldsymbol{C}_{t, i}^{(K-1)}\right] \\
& \boldsymbol{H}_{m i}=\operatorname{diag}\left\{\boldsymbol{H}_{m i}^{(0)}, \boldsymbol{H}_{m i}^{(1)}, \ldots, \boldsymbol{H}_{m i}^{(K-1)}\right\} \\
& \boldsymbol{C}_{f, i}=\operatorname{diag}\left\{\boldsymbol{c}_{f, i}^{(0)}, \boldsymbol{c}_{f, i}^{(1)}, \ldots, \boldsymbol{c}_{f, i}^{(K-1)}\right\} \\
& \boldsymbol{x}_{i}=\left[x_{i}^{(0)}, x_{i}^{(1)}, \ldots, x_{i}^{(K-1)}\right]^{T}
\end{aligned}
$$

Finally, for clarity of presentation, letting $\boldsymbol{H}_{m i}=\boldsymbol{C}_{t, i} \boldsymbol{H}_{m i} \boldsymbol{C}_{f, i}$, which is a $\left(N_{f} N_{t} \times K\right)$ matrix, we can rewrite (8) as

$$
\boldsymbol{y}_{m}=\sum_{i=0}^{2} \boldsymbol{H}_{m i} \boldsymbol{x}_{i}+\boldsymbol{n}_{m}, m=0,1,2
$$

As discussed previously, a BS may detect a part of intercell users (Class-1 intercell users), which are chosen based on a pre-defined threshold $\lambda$. In this case, we can express $\boldsymbol{x}_{i}$ of the symbols transmitted by the intercell users in cell $i$ as

$$
\boldsymbol{x}_{i}=\left[\left(\boldsymbol{x}_{m i}^{(1)}\right)^{T},\left(\boldsymbol{x}_{m i}^{(2)}\right)^{T}\right]^{T}, i \neq m
$$

where $\boldsymbol{x}_{m i}^{(1)}$ and $\boldsymbol{x}_{m i}^{(2)}$ correspond to the Class- 1 and Class-2 intercell users in cell $i$. Accordingly, the channel matrix $\mathcal{H}_{m i}$ can be written as

$$
\boldsymbol{H}_{m i}=\left[\boldsymbol{\mathcal { H }}_{m i}^{(1)}, \boldsymbol{H}_{m i}^{(2)}\right], m \neq i
$$

in correspondence with $\boldsymbol{x}_{m i}^{(1)}$ and $\boldsymbol{x}_{m i}^{(2)}$. Then, using (11) and (12), we can reformulate (10) as

$$
\boldsymbol{y}_{m}=\sum_{i=0}^{2} \tilde{\boldsymbol{H}}_{m i} \tilde{\boldsymbol{x}}_{i}+\boldsymbol{n}_{m}, m=0,1,2
$$

where, when $i=m$,

$$
\begin{aligned}
& \tilde{\boldsymbol{H}}_{m m}=\left[\boldsymbol{\mathcal { H }}_{m m}, \boldsymbol{H}_{m a}^{(1)}, \boldsymbol{H}_{m b}^{(1)}\right], a \neq b \neq m \\
& \tilde{\boldsymbol{x}}_{m}=\left[\boldsymbol{x}_{m}^{T},\left(\boldsymbol{x}_{m a}^{(1)}\right)^{T},\left(\boldsymbol{x}_{m b}^{(1)}\right)^{T}\right]^{T}, a \neq b \neq m
\end{aligned}
$$

and, when $i \neq m$,

$$
\tilde{\boldsymbol{H}}_{m i}=\mathcal{H}_{m i}^{(2)}, \tilde{\boldsymbol{x}}_{i}=\boldsymbol{x}_{m i}^{(2)}
$$

Note that, in (15), $\tilde{\boldsymbol{x}}_{m}$ contains the data symbols of the intracell users and that of the Class-1 intercell users of the two neighboring cells. By contrast, in (16), $\tilde{x}_{i}$ only contains the symbols of the Class- 2 intercell users of cell $i$.

In the following MUD schemes considered, the intracell user symbols are detected based on (13) by treating the Class-1 intercell user symbols as useful information. The dimension of $\tilde{\boldsymbol{x}}_{m}$ is denoted as $\left(K_{m} \times 1\right)$, which is determined by the threshold $\lambda$. Particularly, $K_{m}=K$ when $\lambda=1$, and $K_{m}=3 K$ when $\lambda=0$. By contrast, the signals from the Class-2 intercell users will be treated as harmful interference.

\section{Decision-Feedback Multiuser Detection}

Recently, a decision-feedback MUD referred to as the RMD/MSMMSE MUD has been proposed in [11], which is capable of achieving the near-optimum BER performance with linearly dependent detection complexity. In this section, we extend the RMD/MS-MMSE MUD [11] proposed for single-cell SDMA and DS-CDMA systems to the multicell TF/MC DS-CDMA systems with or without BS cooperation. Let us first consider the RMD/MS-MMSE MUD for the multicell TF/MC DS-CDMA systems without BS cooperation.

\section{A. RMD/MS-MMSE MUD without BS cooperation}

It is well-known that, in multicell wireless systems, ICI may severely degrade the achievable performance. Hence, it is crucial to manage appropriately the signals received from other cells. From [12], we have learnt that, when ICI is strong, a SIC-based decoder is capable of achieving the capacity bound. Therefore, in this contribution, we propose and study the SIC-type detection schemes, which motivate to detect the intracell users by also detecting the Class- 1 intercell users, whose interference on the intracell users is canceled based on the SIC principles. Specifically, we extend the RMD/MS-MMSE MUD proposed in [11] to the multicell TF/MC DS-CDMA systems, where both the intracell and Class-1 intercell users are detected. However, here the Class-1 intercell users are detected only for the purpose of the intracell users' BER performance enhancement, as no BS cooperation is considered in this section. Their information will be thrown away after the detection. Additionally, we note that, when both the intracell users and Class-1 intercell users are simultaneously detected, the TF/MC DS-CDMA system possibly become heavily overloaded, meaning that the number of users detected is much higher than the system's degrees of freedom [11]. Our studies in this contribution will show that the RMD/MS-MMSE MUD is a high-efficiency scheme for application in heavily overloaded scenarios.

According to [11], the RMD/MS-MMSE MUD for the multicell TF/MC DS-CDMA system can be described as follows.

Initialization: For $m=0,1,2$, the number of intracell users plus Class-1 intercell users, $K_{m}$, is determined based on a pre-set value for $\lambda$; The initial observation vector $\boldsymbol{y}_{m}[0]$ and the weight matrix of MMSE MUD $\boldsymbol{W}_{m}[0]$ are formed as

$$
\begin{aligned}
& \boldsymbol{y}_{m}[0]=\boldsymbol{y}_{m}=\sum_{i=0}^{2} \tilde{\mathcal{H}}_{m i} \tilde{\boldsymbol{x}}_{i}+\boldsymbol{n}_{m}, \\
& \boldsymbol{W}_{m}[0]=\left(\boldsymbol{R}_{y, m}[0]\right)^{-1} \boldsymbol{R}_{y x, m}[0],
\end{aligned}
$$

where $\boldsymbol{R}_{y, m}[0]$ is the auto-correlation matrix of $\boldsymbol{y}_{m}[0]$ and $\boldsymbol{R}_{y x, m}[0]$ is the cross-correlation matrix between $\boldsymbol{y}_{m}[0]$ and $\tilde{\boldsymbol{x}}_{m}$, which can be expressed as

$$
\begin{aligned}
& \boldsymbol{R}_{y, m}[0]=\sum_{i=0}^{2} \tilde{\mathcal{H}}_{m i} \tilde{\mathcal{H}}_{m i}^{H}+\sigma^{2} \boldsymbol{I}=\sum_{i=0}^{2} \boldsymbol{\mathcal { H }}_{m i} \boldsymbol{\mathcal { H }}_{m i}^{H}+\sigma^{2} \boldsymbol{I}, \\
& \boldsymbol{R}_{y x, m}[0]=\tilde{\mathcal{H}}_{m m} .
\end{aligned}
$$

Additionally, the channel matrix is initialized as

$$
\tilde{\mathcal{H}}_{m i}[0]=\tilde{\mathcal{H}}_{m i},
$$

where $\tilde{\boldsymbol{H}}_{m i}$ is given in (14) or (16).

Detection: During stage $s=1,2, \ldots$, where $s \leq K_{m}$, the following operations are carried out until all the $K$ intracell users are detected:

a) Forming decision variable vector:

$$
\boldsymbol{z}_{m}[s]=\Re\left\{\left(\boldsymbol{W}_{m}[s-1]\right)^{H} \boldsymbol{y}_{m}[s-1]\right\},
$$

where $\Re\{a\}$ denotes the real part of the variable $a$.

b) Determining and detecting the most reliable user: For the $K_{m}-s+1$ users having not been detected, their reliabilities are measured by the magnitudes of the posteriori log-likelihood ratios (LLRs), expressed as [11]

$$
\begin{aligned}
L_{m}^{(k)}= & \left(1-\left(\tilde{\boldsymbol{h}}_{m m}^{(k)}[s-1]\right)^{H} \boldsymbol{R}_{y, m}^{-1}[s-1] \tilde{\boldsymbol{h}}_{m m}^{(k)}[s-1]\right)^{-1} \\
& \times\left|z_{m}^{(k)}\right|, \quad k=1,2, \ldots, K_{m}-s+1
\end{aligned}
$$

where $\tilde{\boldsymbol{h}}_{m m}^{(k)}[s-1]$ is the $k$ th column of $\tilde{\boldsymbol{H}}_{m m}[s-1]$. Then, the most reliable user is identified, whose decision variable is expressed as $\tilde{z}_{m}[s]$. Correspondingly, the symbol transmitted by the most reliable user is detected as $\hat{x}_{m}[s]=\operatorname{sgn}\left\{\tilde{z}_{m}[s]\right\}$.

c) Interference Cancellation:

$$
\boldsymbol{y}_{m}[s]=\boldsymbol{y}_{m}[s-1]-\tilde{\boldsymbol{h}}_{m m} \hat{x}_{m}[s],
$$

where $\tilde{\boldsymbol{h}}_{m m}$ is the column in $\tilde{\mathcal{H}}_{m m}[s-1]$ corresponding to the most reliable user detected. 
d) Update:

$$
\begin{aligned}
& \tilde{\boldsymbol{H}}_{m m}[s]=\tilde{\mathcal{H}}_{m m}[s-1] \boldsymbol{P}_{m}[s], \\
& \boldsymbol{R}_{y, m}[s]=\boldsymbol{R}_{y, m}[s-1]-\tilde{\boldsymbol{h}}_{m m} \tilde{\boldsymbol{h}}_{m m}^{H}, \\
& \boldsymbol{R}_{y x, m}[s]=\tilde{\mathcal{H}}_{m m}[s], \\
& \boldsymbol{W}_{m}[s]=\left(\boldsymbol{R}_{y, m}[s]\right)^{-1} \boldsymbol{R}_{y x, m}[s],
\end{aligned}
$$

where $\boldsymbol{P}_{m}[s]$ denotes a permutation matrix obtained from $\boldsymbol{I}_{K}$ after removing its $s$ columns corresponding to the $s$ users having been detected so far.

\section{B. RMD/MS-MMSE MUD with Data Exchange and Combine}

In the previous scheme, a BS detects the Class- 1 intercell users in addition to the intracell users, in order to mitigate the strong ICI. However, as there is no cooperation among the BSs, intensive MUI exists in the detection, which significantly degrades the detection performance. Furthermore, without BS cooperation, the information gained from detection of Class-1 intercell users cannot be exploited by the other cells. In this section, we improve the RMD/MS-MMSE MUD considered in Section III-A by introducing BS cooperation achieved with the aid of data exchange among the BSs.

To be more specific, at a detection stage, each BS detects the most reliable user, which may be an intracell user or a Class- 1 intercell user. Then, the detected symbols of the three cells are interchanged via the backhaul-link. We assume that data exchange among the BSs is free of errors. After the data exchange, further decisions about the symbols are required, as the detected symbols may belong to one, two or three users. Therefore, after data exchange, each BS will first check whether the detected symbols belong to the same user. If there are two or three symbols belonging to the same user, the BS then combines these symbols based on the principle of majority voting (MV).

In contrast to the systems without BS cooperation, where each user is only detected by its BS, in the proposed scheme with data exchange, detecting one user can make use of the information provided by three BSs, which introduces Macro-diversity and, hence, improves the detection performance. Below we state the algorithm for the RMD/MSMMSE MUD with data exchange. Note that, in comparison with the RMD/MS-MMSE MUD without BS cooperation, as described in Section III-A, the initialization for the RMD/MS-MMSE MUD with BS cooperation is the same, which is hence omitted.

For BS $m=0,1$ or 2 , after the initialization, the multi-stage detection is operated as follows.

Detection: During stage $s=1,2, \ldots, K_{m}, \ldots$, the following operations are executed.

a) Forming decision variable:

$$
\boldsymbol{z}_{m}[s]=\Re\left\{\left(\boldsymbol{W}_{m}[s-1]\right)^{H} \boldsymbol{y}_{m}[s-1]\right\},
$$

b) Determining and detecting the most reliable user: For the users having not been detected, their reliabilities are computed according to (21). Then, the most reliable user is identified, whose decision variable is expressed as $\tilde{z}_{m}[s]$. Correspondingly, the estimated symbol can be expressed as $\hat{x}_{m}[s]=\operatorname{sgn}\left\{\tilde{z}_{m}[s]\right\}$.

c) Data Exchange: BS $m$ sends $\hat{x}_{m}[s]$ to the other two BSs. Simultaneously, it receives $\hat{x}_{n}[s](n=0,1,2 ; n \neq m)$ from these two BSs.

d) Data Combining: After receiving the detected symbols from the other two BSs, BS $m$ checks whether $\hat{x}_{m}[s]$ and $\hat{x}_{n}[s](n=$ $0,1,2 ; n \neq m$ ) belong to the same user. There are three cases to consider.

CASE 1: The three most reliable users detected by the three BSs are the same user. In this case, the final estimated symbol of BS $m$ are given by $\hat{x}_{m_{0}}^{\prime}[s]=\operatorname{sgn}\left\{\hat{x}_{0}[s]+\hat{x}_{1}[s]+\hat{x}_{2}[s]\right\}$ by the MV principle as well as $\hat{x}_{m_{1}}^{\prime}[s]=\hat{x}_{m_{2}}^{\prime}[s]=0$. Here, $m_{0}, m_{1}$ and $m_{2}$ are the temporally indices for the possible users detected by BS $m$.

CASE 2: BS $i$ and $j$ detect the same most reliable user, while BS $k$ detects a different one, here $(i, j, k=0,1,2 ; i \neq j \neq k)$. In this case, the estimated symbol of the user detected by BSs $i$ and $j$ is $\hat{x}_{m_{0}}^{\prime}[s]=\operatorname{sgn}\left\{\hat{x}_{i}[s]+\hat{x}_{j}[s]\right\}$. However, if $\hat{x}_{m_{0}}^{\prime}[s]=0$, we assign $\hat{x}_{m_{0}}^{\prime}[s]$ a symbol randomly selected from $\hat{x}_{i}[s]$ and $\hat{x}_{j}[s]$. Finally, we set $\hat{x}_{m_{1}}^{\prime}[s]=0$ and $\hat{x}_{m_{2}}^{\prime}[s]=\hat{x}_{k}[s]$.
CASE 3: The three most reliable users detected by the three BSs are different users. Then, BS $m$ sets $\hat{x}_{m_{i}}^{\prime}[s]=\hat{x}_{i}[s](i=0,1,2)$.

e) Interference Cancellation:

$$
\boldsymbol{y}_{m}[s]=\boldsymbol{y}_{m}[s-1]-\sum_{n=0, \hat{x}_{m_{n}}^{\prime}[s] \neq 0}^{2} \tilde{\boldsymbol{h}}_{m n} \hat{x}_{m_{n}}^{\prime}[s],
$$

where, when $\hat{x}_{m_{n}}^{\prime}[s] \neq 0, \tilde{\boldsymbol{h}}_{m n}$ is the column in $\tilde{\mathcal{H}}_{m}[s-1]$ corresponding to the detected user $m_{n}$, here $\tilde{\mathcal{H}}_{m}[s-1]$ is the integrated channel matrix expressed as

$$
\tilde{\mathcal{H}}_{m}[s-1]=\left[\tilde{\mathcal{H}}_{m m}[s-1], \tilde{\mathcal{H}}_{m i}[s-1], \tilde{\mathcal{H}}_{m j}[s-1]\right]
$$

the dimension of which is $\left(N_{f} N_{t} \times 3 K\right)$.

f) Update:

$$
\begin{aligned}
& \tilde{\mathcal{H}}_{m}[s]=\tilde{\boldsymbol{H}}_{m}[s-1] \boldsymbol{P}_{m}[s], \\
& \tilde{\mathcal{H}}_{m m}[s]=\tilde{\mathcal{H}}_{m}[s] \boldsymbol{Q}_{m}[s], \\
& \boldsymbol{R}_{y, m}[s]=\boldsymbol{R}_{y, m}[s-1]-\sum_{n=0}^{2}\left(\hat{x}_{m n}^{\prime}[s] \times \tilde{\boldsymbol{h}}_{m n} \tilde{\boldsymbol{h}}_{m n}^{H}\right), \\
& \boldsymbol{R}_{y x, m}[s]=\tilde{\boldsymbol{H}}_{m m}[s], \\
& \boldsymbol{W}_{m}[s]=\left(\boldsymbol{R}_{y, m}[s]\right)^{-1} \boldsymbol{R}_{y x, m}[s],
\end{aligned}
$$

where $\boldsymbol{P}_{m}[s]$ is a permutation matrix obtained from a $(3 K \times 3 K)$ identity matrix $\boldsymbol{I}_{3 K}$ after setting the columns corresponding to the detected users at stage $s$ to zero vectors, while $\boldsymbol{Q}_{m}[s]$ is a $\left(3 K \times K_{m}\right)$ matrix, which is introduced to obtain $\tilde{\mathcal{H}}_{m m}[s]$ from $\tilde{\mathcal{H}}_{m}[s]$ and can be expressed as

$$
\boldsymbol{Q}_{m}[s]=[\boldsymbol{I}, \mathbf{0}]^{T},
$$

where $\boldsymbol{I}$ is a $\left(K_{m} \times K_{m}\right)$ identity matrix and $\mathbf{0}$ is a $\left(K_{m} \times\left(3 K-K_{m}\right)\right)$ zero matrix.

\section{Performance Results}

In this section, we investigate and compare the error performance of the multicell TF/MC DS-CDMA systems employing binary phaseshift keying (BPSK) modulation using random spreading codes. The impacts of ICI factor (ICIF), and system load are explored. Note that, in the figures, for simplicity, RMD-MMSE is for RMD/MSMMSE MUD without BS cooperation, while RMD-MMSE-DEC is for RMD/MS-MMSE MUD with data exchange and combining.

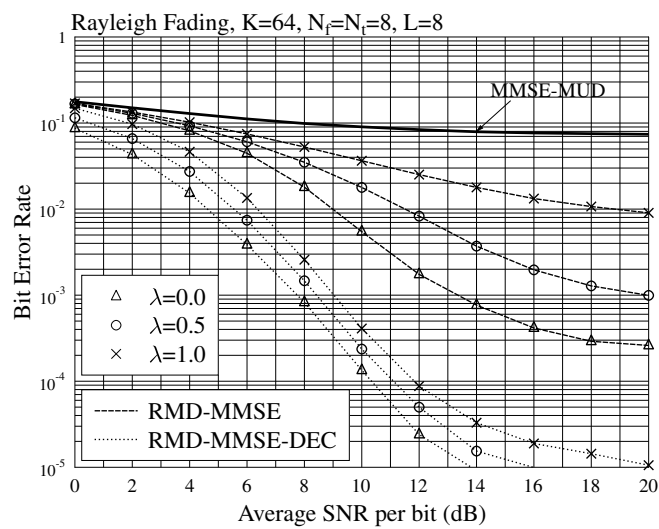

Fig. 3. BER performance of the multicell TF/MC DS-CDMA systems employing RMD/MS-MMSE MUD with or without BS cooperation, when different ICIFs are considered.

Fig. 3 shows the impact of the ICIF of $\lambda$ on the achievable BER performance. In this figure, the BER performance of the linear MMSEMUD without BS cooperation is also provided as a benchmark. From the results, explicitly, we can observe that the RMD/MS-MMSE MUD significantly outperforms the MMSE-MUD and the RMD/MS-MMSE MUD with BS cooperation significantly outperforms the RMD/MSMMSE MUD without employing BS cooperation. 
Considering the impact of the ICIF, as seen in Fig. 3, when $\lambda=1$, implying that each BS only detects its intracell users, the achievable BER performance of the RMD/MS-MMSE MUD (both with and without BS cooperation) is worse than that of the RMD/MS-MMSE MUD associated with an ICIF $\lambda<1$, which explains that every BS detects a part of the intercell users in addition to its intracell users. Finally, when $\lambda=0$, corresponding to the case that each BS detects all the users in the system, the best BER performance is achieved. However, the above-mentioned performance improvement is attained at a cost of higher detection complexity due to the increased number of users detected by each BS. Hence, in practice, depending on the complexity affordable, an appropriate $\lambda$ value for the ICIF may be used, in order to attain a good tradeoff between BER performance and complexity.

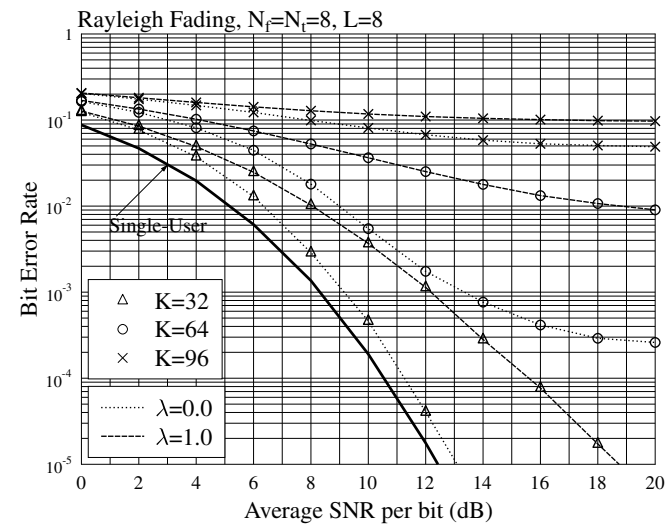

(a) RMD/MS-MMSE MUD without BS cooperation.

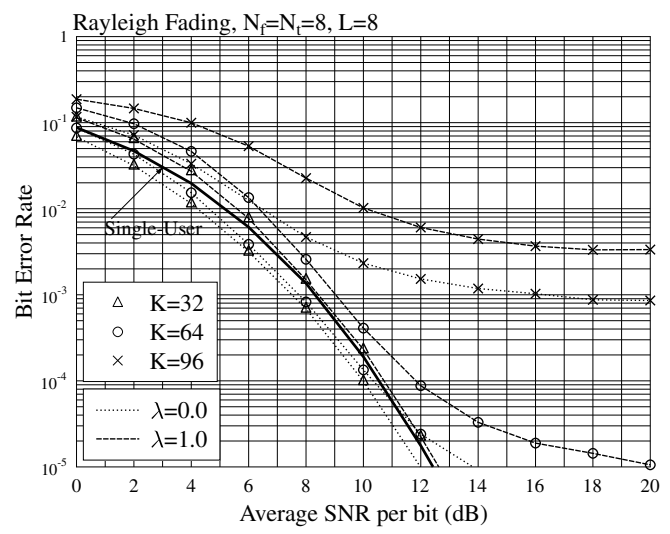

(b) RMD/MS-MMSE MUD with data exchange and combining

Fig. 4. BER performance of the multicell TF/MC DS-CDMA systems employing the RMD/MS-MMSE MUD with or without BS cooperation, when the systems are under-load, full-load or overload.

In Fig. 4, we investigate the effects of system loads on the achievable BER performance of RMD/MS-MMSE MUD. Specifically, the cases of $K=32=0.5 N_{t} N_{f}, K=64=N_{t} N_{f}$ and $K=96=1.5 N_{t} N_{f}$ users per cell are considered, which correspond to the scenarios of under-load, full-load and overload, respectively. Additionally, the single-user BER performance in a single-cell is included as a benchmark. From the results of Fig. 4 (a), where the RMD/MS-MMSE without BS cooperation is considered, when $\lambda=1$, we can observe that, due to the ICI, even the BER performance of the under-load system is much worse than the single-user bound. Furthermore, error floor appears very quickly for the full-load and overload cases. By contrast, when $\lambda=0$, benefit from the ICI mitigation is obvious, great performance improvement can be observed for the under-load and fullload cases, in comparison with the cases with $\lambda=1$. However, the BER performance of the over-load system is still very poor, due to the severe MUI and ICI.
By contrast, as shown in Fig. 4 (b), when the RMD/MS-MMSE MUD employs the BS cooperation supported by data exchange and combining, the BER performance is significantly improved, when compared with those depicted in Fig. 4 (a). Specifically, when $\lambda=1$, we observe the BER performance of the under-load system tends to the single-user bound, while that of the full-load and overload systems gradually ceases from improving as the SNR increases, which is due to the ICI. Comparatively, when $\lambda=0$, we observe that the BER performance of both the under-load and full-load systems is even better than the single-user bound, owing to the macro-diversity gain attained via data exchange and combining among the BSs. Note that, in $[7,10]$, the schemes considered have the (single-cell) single-user bounds as their upper bounds. Furthermore, from Fig. 4 (b), we can see that the RMD/MS-MMSE MUD works well in the systems of heavily overloaded, particularly, when the SNR is relatively low, which is practically desirable.

In conclusions, We have proposed the RMD/MS-MMSE MUDs for the multicell TF/MC DS-CDMA systems with or without BS cooperation. Our studies show that employing the BS cooperation by exchanging hard-decision data significantly enhances the capability of the RMD/MS-MMSE MUDs, resulting in much better BER performance than the RMD/MS-MMSE MUDs without BS cooperation. The achievable BER performance of the RMD/MS-MMSE MUDs no matter whether BS cooperation is employed or not - can also be improved, if each of the BSs tries to detect a part of intercell users in addition to detecting its intracell users. Furthermore, our studies show that the RMD/MS-MMSE MUDs are highly efficient MUDs. They are capable of attaining promising BER performance, even when each of the cells is heavily overloaded. Additionally, they are low-complexity MUD schemes, as analyzed in [11].

\section{ACKNOWLEDGEMENT}

This work was supported in part by the National Basic Research Program of China (973 Program, Grant No. 2010CB731803), the National Natural Science Foundation of China (Grant No. 60921001 and 61071072).

\section{REFERENCES}

[1] L.-L. Yang and L. Hanzo, "Multicarrier DS-CDMA: a multiple access scheme for ubiquitous broadband wireless communications," IEEE Comm. Mag., vol. 41, no. 10, pp. 116 - 124, Oct 2003.

[2] L.-L. Yang, Multicarrier Communications. Chichester, United Kingdom: John Wiley, 2009.

[3] P. Pan, L.-L. Yang, and Y. Zhang, "Time-frequency iterative multiuser detection in time-frequency-domain spread multicarrier DS-CDMA systems over nakagami-m fading channels," European Trans. on Telecomm., vol. 22, no. 1, pp. 2-13, 2011.

[4] S. Verdú, Multiuser Detection. Cambridge University Press, 1998.

[5] S. Karimifar and J. Cavers, "Achieving high-capacity narrowband cellular systems by means of multicell multiuser detection," IEEE Trans. on Veh. Tech., vol. 57, no. 2, pp. 945 -953, Mar 2008.

[6] Z. Haas and C.-P. Li, "The multiply-detected macrodiversity scheme for wireless cellular systems," IEEE Trans. on Veh. Tech., vol. 47, no. 2, pp. $506-530$, may 1998.

[7] T. Mayer, H. Jenkac, and J. Hagenauer, "Turbo base-station cooperation for intercell interference cancellation," in Proc. IEEE ICC, Jun 2006, pp. $4977-4982$

[8] S. Khattak, W. Rave, and G. Fettweis, "Distributed iterative multiuser detection through base station cooperation," EURASIP J. Wirel. Commun. Netw., vol. 2008, pp. 17:1-17:15, Jan 2008. [Online]. Available: http://dx.doi.org/10.1155/2008/390489

[9] S. Bavarian and J. Cavers, "Reduced-complexity belief propagation for system-wide MUD in the uplink of cellular networks," IEEE JSAC, vol. 26, no. 3, pp. $541-549$, Apr. 2008.

[10] P. Li and R. de Lamare, "Parallel multiple candidate interference cancellation with distributed iterative multi-cell detection and base station cooperation," in 2012 Int. ITG Workshop on Smart Antennas (WSA), Mar 2012, pp. $92-96$.

[11] L.-L. Yang, "Receiver multiuser diversity aided multi-stage minimum mean-square error detection for heavily loaded DS-CDMA and SDMA systems," IEEE Trans. on Comm., vol. 58, no. 12, pp. 3397 -3404, Dec. 2010.

[12] H. Sato, "The capacity of the gaussian interference channel under strong interference," IEEE Trans. on Inf. Theory, vol. 27, no. 6, pp. $786-788$, Nov. 1981. 